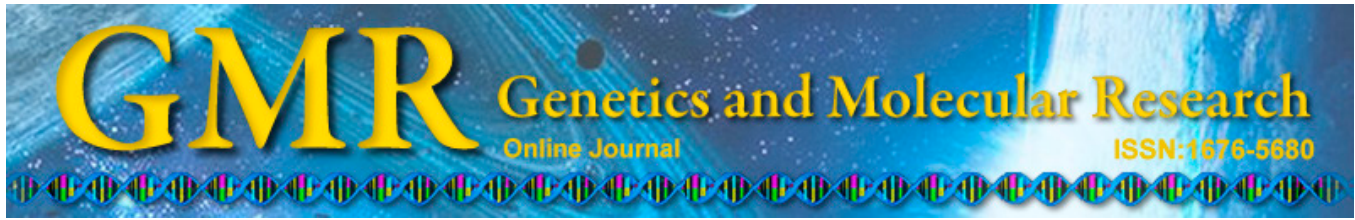

\title{
Application value of multislice spiral computed tomography angiography in the evaluation of renal artery variation in living donor kidney transplantation
}

\author{
X.Y. Zhao ${ }^{1}$, J. Tian' 1 , Y.H. Ru' ${ }^{1}$, B. Sun ${ }^{1}$, C.F. Sun ${ }^{1}$, A.M. Zhang ${ }^{2}$ and Y.H. Shao \\ 'Department of Nuclear Medicine, Jinan Military General Hospital, Jinan, China \\ ${ }^{2}$ Department of Urology, Jinan Military General Hospital, Jinan, China \\ Corresponding author: Y.H. Shao \\ E-mail: yahuishaocn@126.com
}

Genet. Mol. Res. 14 (1): 314-322 (2015)

Received April 28, 2014

Accepted October 24, 2014

Published January 23, 2015

DOI http://dx.doi.org/10.4238/2015.January.23.5

\begin{abstract}
This study aims to investigate the accuracy and value of multislice spiral computed tomography (MSCT) angiography in the evaluation of renal artery variation in living donor kidney transplantation. Two hundred seventy-three kidney transplantation donors underwent preoperative MSCT scanning. Two doctors determined the running direction and variation of the renal artery through joint analysis of the preoperative original MSCT image and the recombination image using the blind reading method, compared the imaging results with the intraoperative results, and evaluated the accuracy and application value of MSCT angiography in the evaluation of renal artery variation in living donor kidney transplantation. CT angiography (CTA) can better show the renal artery and its variation. A total of 52 accessory renal arteries were found in the 273 kidney transplant operations, whereas 55 accessory renal arteries were found in preoperative MSCT. Four accessory renal arteries indicated in the MSCT were not found during the operation, and one accessory renal artery found during the operation
\end{abstract}


was not indicated in the preoperative MSCT. The sensitivity, specificity, positive predictive value, negative predictive value, and accuracy of MSCT in the diagnosis of accessory renal arteries were 98.1, 98.2, 92.7, 99.5 , and $98.2 \%$, respectively. MSCT angiography can sensitively and accurately show the renal artery and its variation in living donor kidney transplantation, and has important clinical value for the formulation of the operative scheme before the transplantation.

Key words: Kidney transplantation; Living donor; CT angiography; Preoperative evaluation; Tomography; Computed radiography

\section{INTRODUCTION}

Kidney transplantation has become the most effective therapeutic measure for the treatment of end-stage renal disease. To solve the problem of kidney shortage, many hospitals implement living kidney transplantation. Living kidney donors can relieve the problem of organ shortage to a great extent (Yap et al., 2010; Zhao et al., 2012; Kute et al., 2012; Balachandran et al., 2012). The aim of living donor kidney transplantation is to ensure the life safety of both the donor and receptor. To ensure the safety of the donor, the choice of the method for excision of the donor kidney is crucial. Preoperative evaluation of the vascular anatomy of the donor kidney is the main basis for donor kidney selection and the formulation of the donor nephrectomy scheme. Renal angiography evaluation mainly includes digital subtraction angiography (DSA), magnetic resonance angiography, color Doppler flow imaging, and multislice spiral computed tomography (MSCT) angiography. After comparing the advantages and limitations of different imaging methods, and combining with the knowledge of the anatomical characteristics and clinical requirements of blood vessels in the donor kidney, most transplantation centers evaluate the anatomical structure and adjacent relation of the blood vessels in the donor kidney by using MSCT, the outcomes of which have attracted extensive attention and recognition (Valastro et al., 2007; Tombul et al., 2008; Anderson et al., 2008; Chai et al., 2008; Singh et al., 2008; Yap et al., 2010). Previous studies reported fewer cases, and the influence of CT scanning and image post-processing technology on the diagnostic accuracy were insufficiently studied. This article investigates the accuracy and clinical application value of MSCT in the evaluation of renal artery variation in living donor kidney transplantation, with intraoperative findings as the gold standard.

\section{MATERIAL AND METHODS}

\section{Clinical data}

We retrospectively analyzed the clinical data of 273 kidney donors, for the living kidney transplantation of their relatives, in the Department of Urology of our hospital in October 2007 to December 2011. The donors underwent preoperative MSCT and had complete intraoperative data; they comprised 186 male and 87 female donors with an average age of $37.7 \pm$ 11.5 years (range, 20-65 years). This study was conducted in accordance with the Declaration of Helsinki and with approval from the Ethics Committee of Jinan Military General Hospital. Written informed consent was obtained from all participants. 


\section{Examination method}

Biograph Sensation 16 (Siemens Medical Systems, Erlangen, Germany) was used for the CT examination. The patients were first subjected to a noncontrast enhanced scan, and to early arterial and late arterial + delayed computed tomography urography (CTU) scanning through the injection of $90 \mathrm{~mL}$ nonionic contrast agent (Ultravist 370; Guangzhou Branch of Bayer Healthcare Co., Ltd., Guangzhou, China) with an iodine content of $37 \mathrm{~g} / 100 \mathrm{~mL}$ into the elbow vein, by using a high-pressure syringe (Stellant; Medrad, Indianola, PA, USA) at an injection rate of $4.0 \mathrm{~mL} / \mathrm{s}$. Delayed imaging of early artery was monitored in real time by using the Bolus Tracking software system at the origin of the abdominal aorta. Ten seconds after the injection began, the monitoring scan was started. Five seconds after the monitoring threshold value reached $40 \mathrm{HU}$ (scanning was prepared for $5 \mathrm{~s}$ ), CT scan was started; early arterial scanning lasted for about 11-12 s, late arterial scanning was started $8 \mathrm{~s}$ after the early arterial scanning was completed, and CTU scanning was started 10-12 min after the late arterial scanning was completed. The patients held their breath in each scanning. The scanning parameters were as follows: tube voltage, $120 \mathrm{kV}$; tube current, $160 \mathrm{mAs}$; late arterial scanning and CTU scanning, $140 \mathrm{mAs}$; collimation, $16 \times 0.75 \mathrm{~mm}$; pitch, 0.75 ; slice, $5.0 \mathrm{~mm}$; and feed/rotation, $9.0 \mathrm{~mm}$. It took $0.5 \mathrm{~s}$ for the pipette to rotate one cycle. The scanning range was from the upper pole of the kidney to the upper edge of the iliac crest, and the lower edge of CTU scanning was the pubic symphysis level. The thin slice reconstruction was implemented with the body soft tissue algorithm, B31f medium smooth filtering function kernel and a slice thickness of 1.0 $\mathrm{mm}$, and data post-processing was done with the volume rendering technique (VRT) by using the Syngo software, maximum intensity projection (MIP), multiplanar reconstruction (MPR), and curved planar reconstruction (CPR).

\section{Image interpretation}

According to the original cross-section, in combination with VRT, MIP, MPR, CPR, and other reconstruction images, two attending physicians analyzed the following contents through blind reading: the opening position and number of accessory renal arteries, the site of their entry into the kidney and their relation with the main renal artery, and the presence of any early renal artery branch. An accessory renal artery refers to a renal artery other than the main renal artery, and can be subdivided into the accessory portal renal artery, accessory upper polar artery, and accessory lower polar artery; an early renal artery branch means that the distance from the branch to the origin of the renal artery is $\leq 1.5 \mathrm{~cm}$. When the two doctors had differing opinions, an agreement was reached through discussion.

\section{Statistical analysis}

The MSCT results were compared by using the SPSS13.0 software to analyze the intraoperative findings, which were taken as the gold standard. The accessory renal artery data from MSCT were compared with the intraoperative results by using the $\chi^{2}$ test, and the sensitivity, specificity, positive predictive value, negative predictive value, and accuracy of MSCT in the evaluation of the anatomical structure of the renal artery were calculated. 


\section{RESULTS}

\section{CTA}

\section{Accessory renal artery}

A total of 79 accessory renal arteries were found in the CTA of the left kidney. There were 1,2 , and 3 accessory renal arteries in 56,7 , and 3 patients, respectively. The opening position of 47 accessory renal arteries was located at the origin of the main renal artery and that of 32 accessory renal arteries was located at the end of the main renal artery. Accessory renal arteries that enter the kidney from the upper pole, hilus, and lower pole accounted for $44.3 \%$ (35/79), 34.2\% (27/79), and 21.5\% (17/79), respectively, of which 15 accessory renal arteries were beyond the plane of the donor kidney. A total of 76 accessory renal arteries were found on the CTA of the right kidney. There were 1, 2, and 3 accessory renal arteries in 61, 5, and 2 patients, respectively. The opening position of 35 accessory renal arteries was located at the origin of the main renal artery and that of 41 accessory renal arteries was located at the end of the main renal artery. Accessory renal arteries that enter the kidney from the upper pole, hilus, and lower pole accounted for $27.6 \%(21 / 76), 32.9 \%(25 / 76)$, and $39.5 \%(30 / 76)$, respectively, of which 14 accessory renal arteries were beyond the plane of the donor kidney. On the basis of the number of donors, accessory renal arteries were found in 109 donors, with an incidence of $39.9 \%$. On the basis of the number of kidneys, accessory renal arteries were found in a total of 134 kidneys, with an incidence of $24.5 \%$. Among the 155 accessory renal arteries, patients with 1,2 , and 3 accessory renal arteries accounted for $75.5,15.5$, and 9.7\%, respectively (Figure 1A-E).

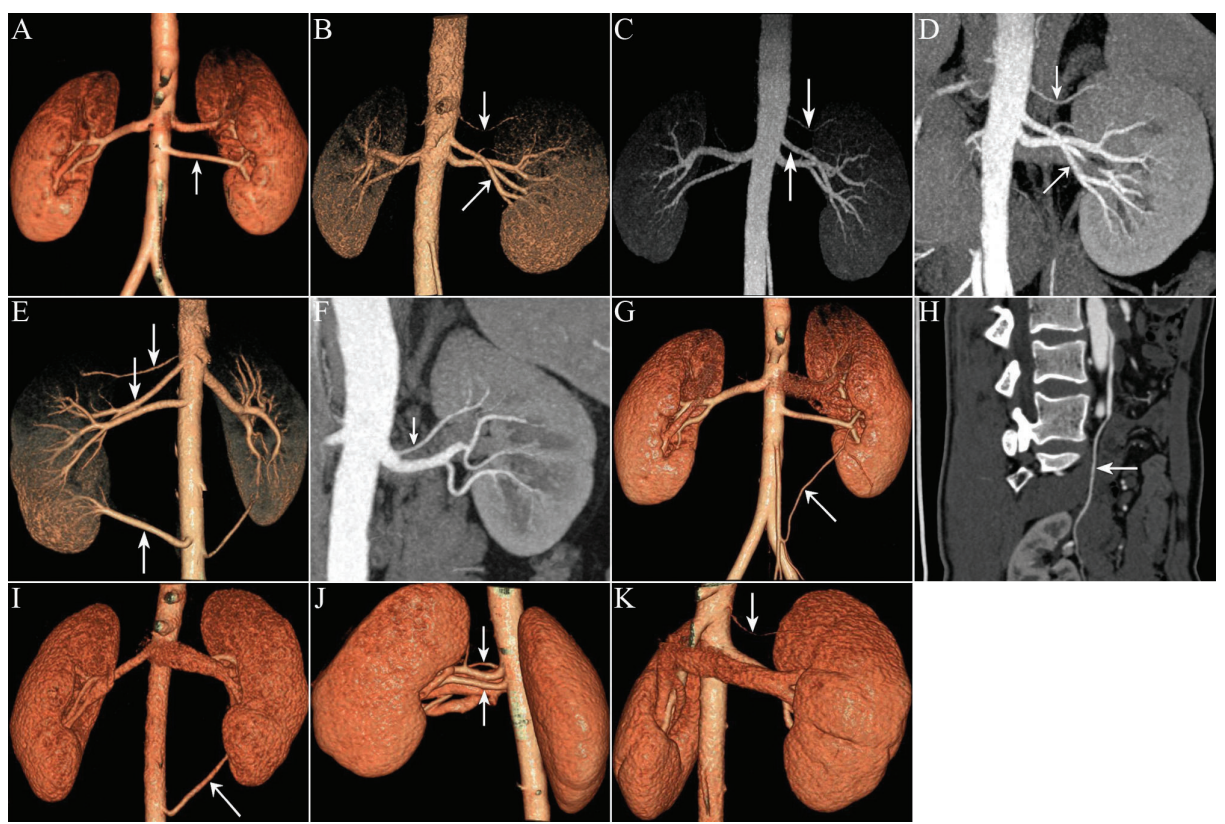

Figure 1. Renal artery and its variation in living donor kidney transplantation indicated by angiography. 


\section{Early branch}

CTA showed 37 and 28 early branches in the left and right kidney, respectively, with an incidence of 13.6 and $10.3 \%$, respectively (Figure $1 \mathrm{~F}$ ).

\section{MSCT (comparison with intraoperative results)}

Two hundred seventy-three donors successfully underwent donor nephrectomy. Fiftytwo accessory renal arteries were found in 273 patients undergoing nephrectomy; 1, 2, and 3 accessory renal arteries were found in 35, 7, and 1 patient, respectively. Preoperative MSCT showed 55 accessory renal arteries, of which 4 accessory renal arteries indicated in MSCT were not found during the operation and 1 accessory renal artery found during the operation was not indicated in preoperative MSCT. The sensitivity, specificity, positive predictive value, negative predictive value, and accuracy of MSCT in the diagnosis of accessory renal arteries were 98.1, 98.2, 92.7, 99.5, and 98.2\%, respectively (Table 1).

Table 1. Comparison of accessory renal arteries diagnosed by MSCT and intraoperative findings (branch).

\begin{tabular}{lcc}
\hline MSCT & \multicolumn{3}{c}{ Intraoperative findings } \\
\cline { 2 - 4 } & Positive & Negative \\
\hline Positive & 51 & 4 \\
Negative & 1 & 217 \\
\hline
\end{tabular}

\section{DISCUSSION}

The preoperative noninvasive investigation of the detailed anatomical structure of the blood vessels in the donor kidney is one of the important bases for selecting which kidney will be taken from the living donor. It also reduces postoperative complications (Dachman et al., 1996; Kuwabara et al., 2006; Raman et al., 2006; Chu et al., 2012; Liefeldt et al., 2012).

MSCT provides a tool for the CT examination of the renal artery, and improves the accuracy and sensitivity of the detection of renal artery variation (Laugharne et al., 2007). However, the MSCT scanning technique and post-processing procedures are not uniform, and this reduces the advantages of the procedure. The selection of enhanced scanning parameters and the reasonable application of post-processing technology are two important steps in renal artery CT imaging. Factors affecting enhanced scanning include the type, amount, and injection speed of the contrast agent; delayed scanning time; structural characteristics of the nephridial tissue itself; and renal function. The total amount of contrast agent that examinees can receive in a short period is limited, and their blood vessels can tolerate only limited pressure from the contrast agent within a short time. Therefore, among the controllable factors, the type, amount, and injection speed of the contrast agent could be made uniform; namely, in most cases, a total of 80-150 mL nonionic contrast agent is used at an injection speed of 3-4 $\mathrm{mL} / \mathrm{s}$, and the main difference lies in the length of the delayed scanning time and the determination of the time point. At present, there are three alternative techniques for selecting the delayed scanning time and determining the time point (Türkvatan et al., 2009; Yap et al., 2010; Koplay et al., 2010; Kim et al., 2011; Kulkarni et al., 2011; Bazeed et al., 2011). 1) Method 1- empirical value method: according to the experience value, the scan is performed in 25 and 
$50 \mathrm{~s}$ to show arteries and veins, respectively. 2) Method 2 - bolus test: the circulation time is measured and determined by using the time density curve. After a bolus injection of $20 \mathrm{~mL}$ contrast agent, the time taken by the contrast agent to travel from the elbow vein to the peak value in the region of interest of the aorta is measured using a corresponding software, and is considered to be the delay time of enhanced scanning. 3) Method 3 - bolus tracking: the delayed scanning time is determined by using a direct contrast agent tracking (automatic trigger) technique, through establishing a trigger threshold value in the region of interest of the aorta. Upon reaching the threshold value, the machine is started automatically and is delayed for 3-5 $\mathrm{s}$; it then automatically triggers the scanning. To avoid the impact of individual differences and the increased radiation dose caused by repeated scanning, the bolus tracking method is more frequently used at present. Various reports provide greatly different trigger threshold settings, which ranges from 50 to $200 \mathrm{HU}$; moreover, there is no detailed description of the effect of different threshold values on the observation of target vessels (Namasivayam et al., 2006; Chai et al., 2008; Petridis et al., 2008; Zamboni et al., 2010). It should be noted that with the increase of the threshold value, the renal vein gradually becomes more obviously developed in the arterial phase scan, which affects the observation of renal arteries, especially the tiny branches. In this study, we adopted the trigger threshold value of $100 \mathrm{HU}$ in the early stage, and then changed it to $40 \mathrm{HU}$ so as to reduce the interference of venography on the observation of arteries, to more clearly show the arterial branches below the arterial segments, and to better predict preoperatively the blood supply area of renal artery branches and accessory renal arteries. These produced satisfactory angiographs; however, the effect of the threshold setting on the target vessel display was not statistically comparatively analyzed in detail, which is also a weakness of this study. This will be further studied and summarized.

In the MSCT examination of renal arteries, the frequently used post-processing techniques include VRT, MIP, MPR, and CPR (Berg et al., 1998). Various post-processing techniques have different imaging characteristics. VRT is characterized by a simple operation, intuitive three-dimensional display of renal artery details, and a clear display of the spatial anatomical relation of the renal artery and its variation; however, it cannot show the internal features of the renal arteries. MIP has a gray level that reflects the relative CT value, produces a reconstruction image similar to conventional angiography, and can clearly show the main renal artery and its main branches; however, its three-dimensional appeal is not as good as that of VRT, and its display of overlapped vessels and structures with similar density is restricted (Figure 1B and C). Thin MIP can avoid the above weaknesses to a certain extent and can better show the overall running direction of blood vessels in the reconstruction image than MPR; however, the layer details of the image are not as well defined as those of MPR (Figure 1D). MPR is a recombination imaging method based on the reconstruction image of the crosssection with a high resolution of density and without overlapping front and back tissues, and it can show the running direction and anatomy of blood vessels from different angles; however, it can show neither the blood vessels beyond the recombination layer in a planar image, nor the extension and running direction of the blood vessels in the image at the same level. Thin MPR and CPR are two special forms of MPR. Thin MPR reduces the cortex interference and more clearly shows the tiny branches. CPR produces the recombination image formed by the profile curve drawn along the vascular center, and can straighten the circuitous blood vessels and show them in the image at the same level. For the anatomical display of the renal artery, with respect to image post-processing, this study showed that a variety of post-processing techniques of MSCT have complementary advantages. A high-quality image of the original cross- 
section is the basis of various post-processing techniques, and VRT and MIP technologies should be preferred. The combined application of these methods can best show the variation of renal arteries and can effectively avoid misjudgment of overlapped vascular branches. This allows distinguishing the suspicious signs and observing the details. The combination of CPR and thin MIP can clearly show the anatomical details of tiny blood vessels and can effectively avoid intraoperative hemorrhage and accidental extrarenal vascular injury caused by misjudgment (Figure $1 \mathrm{G}$ and $\mathrm{H}$ ). It should be emphasized that the image of the original cross-section in each patient should be routinely and carefully observed to further avoid missed diagnosis of some renal arteries with tiny variations.

During kidney transplantation, preoperative image evaluation can accurately show the anatomy and variation of the renal arteries, and not only can provide important reference for the selection of the operation method but also effectively prevents the occurrence of complications, such as hemorrhage, renal infarction, ureteral necrosis, and urine leakage, caused by misjudgment of the vascular anatomy. Renal artery variation mainly includes the accessory renal arteries and early renal artery branches. In the subjects of this study, in accordance with the number of donors, accessory renal arteries were found in 109 donors, with an incidence of $39.9 \%$. On the basis of the number of kidneys, accessory renal arteries were found in a total of 134 kidneys, with an incidence of $24.5 \%$. Among the 155 accessory renal arteries, patients with 1,2 , and 3 accessory renal arteries accounted for $75.5,15.5$, and 9.7\%, respectively. Compared with the incidence of accessory renal arteries in previous reports, the incidence of accessory renal arteries calculated in accordance with the number of donors in this study was higher than the 24-28\% reported in the literature (Berg et al., 1998; Chai et al., 2008; Yap et al., 2010; Zamboni et al., 2010; Kulkarni et al., 2011). The findings of this study show that the sensitivity, specificity, positive predictive value, negative predictive value, and accuracy of MSCT in the diagnosis of renal arteries were 98.1, 98.2, 92.7, 99.5, and 98.2\%, respectively, and were similar to those in the literature (Rajamahanty et al., 2005; Rastogi et al., 2006; Chai et al., 2008; Kim et al., 2011; Kulkarni et al., 2011). The data provided by MSCT can fully meet the requirements of surgery, and this further verifies the accuracy and clinical value of MSCT in the evaluation of renal vascular structure. Therefore, MSCT can be used to replace DSA as the standard examination method for the evaluation of preoperative vascular structure in the donor kidney (Chai et al., 2008; Yap et al., 2010; Chu et al., 2012). At the same time, previous studies have shown that some accessory renal arteries are located beyond the kidney plane, which has not been reported in detail previously, and this has a guiding significance for MSCT scanning and the surgical operation range (Figure 1I). However, it should be noted that this study takes intraoperative findings as the gold standard, and surgical operation almost excludes kidneys with a complex vascular structure; therefore, MSCT findings cannot be contrasted one by one, which is also the relative weakness of this study.

In this study, MSCT misdiagnosed five accessory renal arteries. The cause may be as follows: 1) in the initial stage, the complex vascular variation was insufficiently understood; 2) because of the machine resolution, operation technique, or individual differences, the blood vessels with tiny diameters were not displayed; 3 ) the knowledge of the diagnostician about the characteristics of different recombination images and his comprehensive application ability was also an important subjective factor. For example, MSCT did not find any small artery entering the kidney from the back of the organ, and showed four accessory renal arteries that were inconsistent with the intraoperative findings (Figure 1J and K).

In conclusion, MSCT can accurately show the anatomical structure of renal vessels, 
can fully meet the requirements of clinical operation, and can be used as the standard method for the evaluation of the preoperative anatomical structure of blood vessels in living donor kidney transplantation.

\section{REFERENCES}

Anderson KM, Lindler TU, Lamberton GR, Baron PW, et al. (2008). Laparoscopic donor nephrectomy: effect of perirenal fat upon donor operative time. J. Endourol. 22: 2269-2274.

Balachandran VP, Aull MJ, Charlton M, Afaneh C, et al. (2012). Kidneys from older living donors provide excellent intermediate-term outcomes after transplantation. Transplantation 94: 499-505.

Bazeed MF, Fooshang FF and Ahmed MA (2011). Low-radiation-dose dual-phase MDCT protocol with split contrast media dose and time optimization: protocol design for renal donors evaluation. Acta Radiol. 52: 927-932.

Berg MH, Manninen HI, Vanninen RL, Vainio PA, et al. (1998). Assessment of renal artery stenosis with CT angiography: usefulness of measurements and densitometric analysis of renal parenchymal enhancement as adjuncts to MIP film reading. J. Comput. Assist. Tomogr. 22: 533-540.

Chai JW, Lee W, Yin YH, Jae HJ, et al. (2008). CT angiography for living kidney donors: accuracy, cause of misinterpretation and prevalence of variation. Korean J. Radiol. 9: 333-339.

Chu LC, Sheth S, Segev DL, Montgomery RA, et al. (2012). Role of MDCT angiography in selection and presurgical planning of potential renal donors. A.J.R. Am. J. Roentgenol. 199: 1035-1041.

Dachman AH, Newmark GM, Mitchell RL and Woodle ES. (1996). Helical CT examination of potential kidney donors. A.J.R. Am. J. Roentgenol. 171: 193-200.

Kim MU, Choi KH, Yang SC, Oh YT, et al. (2011). Prospective evaluation of the accuracy of MDCT angiography for living kidney donor. Korean J. Urol. 52: 124-129.

Koplay M, Onbas O, Alper F, Gulcan E, et al. (2010). Multiple renal arteries: variations demonstrated by multidetector computed tomography angiography. Med. Princ. Pract. 19: 412-414.

Kulkarni S, Emre S, Arvelakis A, Asch W, et al. (2011). Multidetector CT angiography in living donor renal transplantation: accuracy and discrepancies in right venous anatomy. Clin. Transplant. 25: 77-82.

Kute VB, Shah PR, Vanikar AV, Gumber MR, et al. (2012). Long-term outcomes of renal transplants from spousal and living-related and other living-unrelated donors: a single center experience. J. Assoc. Physicians India 60: 24-27.

Kuwabara M, Narumi Y, Takahashi S, Sato Y, et al. (2006). Noninvasive method using multidetector CT for calculating the relative blood supply ratio of duplicated renal arteries in renal donors. Radiat. Med. 24: 195-201.

Laugharne M, Haslam E, Archer L, Jones L, et al. (2007). Multidetector CT angiography in live donor renal transplantation: experience from 156 consecutive cases at a single centre. Transpl. Int. 20: 156-166.

Liefeldt L, Klüner C, Glander P, Giessing M, et al. (2012). Non-invasive imaging of living kidney donors: intraindividual comparison of multislice computed tomography angiography with magnetic resonance angiography. Clin. Transplant. 26: E412-E417.

Namasivayam S, Small WC, Kalra MK, Torres WE, et al. (2006). Multidetector-row CT angiography for preoperative evaluation of potential laparoscopic renal donors: how accurate are we? Clin. Imaging 30: 120-126.

Petridis A, Papachristodoulou A, Geroukis T, Fouzas I, et al. (2008). Preoperative evaluation of living kidney donors with multidetector computed tomography angiography. Transplant. Proc. 40: 3137-3141.

Rajamahanty S, Simon R, Edye M, Butt K, et al. (2005). Accuracy of three-dimensional CT angiography for preoperative vascular evaluation of laparoscopic living renal donors. J. Endourol. 19: 339-341.

Raman SS, Pojchamarnwiputh S, Muangsomboon K, Schulam PG, et al. (2006). Utility of 16-MDCT angiography for comprehensive preoperative vascular evaluation of laparoscopic renal donors. A.J.R. Am. J. Roentgenol. 186: 1630-1638.

Rastogi N, Sahani DV, Blake MA, Ko DC, et al. (2006). Evaluation of living renal donors: accuracy of three-dimensional 16-section CT. Radiology 240: 136-144.

Singh PB, Goyal NK, Kumar A, Dwivedi US, et al. (2008). Renal transplantation using live donors with vascular anomalies: a salvageable surgical challenge. Saudi J. Kidney Dis. Transpl. 19: 554-558.

Tombul ST, Aki FT, Gunay M, Inci K, et al. (2008). Preoperative evaluation of hilar vessel anatomy with 3-D computerized tomography in living kidney donors. Transplant. Proc. 40: 47-49.

Türkvatan A, Akinci S, Yildiz S, Olçer T, et al. (2009). Multidetector computed tomography for preoperative evaluation of vascular anatomy in living renal donors. Surg. Radiol. Anat. 31: 227-235.

Valastro M, Veroux M, Macarone M, Cappello D, et al. (2007). Multi-detector row CT scanner angiography in the evaluation of living kidney donors. Chir. Ital. 59: 337-341.

Genetics and Molecular Research 14 (1): 314-322 (2015)

CFUNPEC-RP www.funpecrp.com.br 
Yap DY, Chu FS, Chu SM, Tam PC, et al. (2010). CT angiography versus conventional digital angiography in preoperative assessment for Chinese living kidney donors. J. Nephrol. 23: 711-716.

Zamboni GA, Romero JY and Raptopoulos VD (2010). Combined vascular-excretory phase MDCT angiography in the preoperative evaluation of renal donors. A.J.R. Am. J. Roentgenol. 194: 145-150.

Zhao X, Shao Y, Wang Y, Tian J, et al. (2012). New normal values not related to age and sex, of glomerular filtration rate by $(99 \mathrm{~m})$ Tc-DTPA renal dynamic imaging, for the evaluation of living kidney graft donors. Hell. J. Nucl. Med. 15: 210-214. 